\title{
Score Normalization in Multimodal Systems using Generalized Extreme Value Distribution
}

Renu Sharma ${ }^{1,2}$

renu@cdac.in

Sukhendu Das ${ }^{2}$

sdas@iitm.ac.in

Padmaja Joshi ${ }^{1}$

padmaja@cdac.in

\author{
${ }^{1}$ Centre for Development of Advanced Computing, Mumbai, India
}

${ }^{2}$ Indian Institute of Technology, Madras, India
In multimodal biometric systems, human identification is performed by fusing information at different levels like sensor-level, feature-level, score-level, rank-level and decision-level. Score level fusion is preferred over other levels of fusion because of its low complexity and sufficient availability of information for fusion. However, the scores obtained from different unimodal systems are heterogeneous in nature. For instance, one classifier gives a similarity measure while another gives a dissimilarity measure. Some classifiers give scores indicating the probability of the input pattern to be a genuine subject. Further, distributions of scores obtained from the individual matchers need not be on the same numerical scale (variance) and location (mean). Hence there is a need of normalization before score-level fusion.

Jain et al. [1] described various score normalization techniques for multimodal systems. Shi et al. [2] introduced Extreme Value Theory (EVT) distribution (based on Generalized Pareto Distribution) in unimodal biometric systems for score normalization. They have used a non-parametric method for modelling the significant part of the genuine distribution and a parametric Generalized Pareto Distribution for modelling the tail part of the genuine distribution. Later on, Scheirer et al.[3] also proposed an EVT-based adaptive score normalization method (W-Score) using the Weibull distribution. The methods proposed in [2, 3] focused on the modelling the tail of the impostor (or genuine [2]) distribution. We assume that genuine scores form the tail of a complete (genuine and imposter combined) distribution, and hence we analyze the tail of the complete distribution by considering only the genuine scores. Generalized Extreme Value (GEV) distribution is used for modelling the genuine scores, and parameters of GEV are estimated using the maximum likelihood estimation (MLE). Score vectors used for the estimation of parameters (training) are termed as probe score vectors (Figure 1). Score normalization of the test score vector (query) is performed using the cumulative density function (CDF) of the GEV distribution formed with the learned parameters. Figure 1 represents the entire architecture of the proposed method for score normalization.

There are two extreme value analysis based approaches [4]: (i) Block Maxima and (ii) Peak over Threshold. Shi et al. [2] method is based on peak over threshold approach. Our proposed method and W-Score [3] technique are based on the block maximum approach for extreme value analysis. W-Score method is an adaptive impostor-centric technique, which uses a single score vector obtained by comparing the input test template (during query or testing) to the enrolled templates. From the score vector, the W-Score method uses only the top impostor scores (excluding the topmost score) to fit a Weibull distribution. In contrast to the W-Score, our method is client-centric, and for modelling the GEV distribution the number of probe samples and their corresponding score vectors are utilized. This process occurs offline. From each score vector, one genuine score and N-1 impostor scores are obtained. A single genuine score is considered as an extreme value in the score vector. A collection of genuine scores form a set of extreme values with respect to the whole set of probe score vectors. If a single probe score vector is considered as a block, genuine scores form a sequence of minimum (or maximum) values. According to the EVT theory [4], minima (or maxima) of sequences is characterized by the GEV distribution, whose $\mathrm{CDF}$ is given as:

$G(x, \mu, \sigma, k)=\left\{\begin{array}{l}\exp \left(-\left(1+k\left(\frac{x-\mu}{\sigma}\right)\right)^{-1 / k}\right) \text { if } k \neq 0 \\ \exp \left(-\exp \left(-\left(\frac{x-\mu}{\sigma}\right)\right)\right) \text { if } k=0\end{array}\right.$

where, $1+k(x-\mu) / \sigma>0$ is such that $1+k x>0 . \mu, \sigma$ and $k$ correspond to the mean, standard deviation and shape parameters of the distribution respectively. So, the genuine data from the probe score vectors are modelled by the GEV distribution and the parameter set (mean, scale and location) is computed by the maximum likelihood estimation method. If $S_{1}, \ldots, S_{M}$ are the $\mathrm{M}$ genuine scores, the loglikelihood function to be maximized, is formulated as:
$L L(\mu, \sigma, k)=-M \log \sigma-\sum_{j}\left[1+\frac{k\left(S_{j}-\mu\right)}{\sigma}\right]^{-1 / k}-\left(\frac{1}{k}+1\right) \sum_{j} \log \left[1+\frac{k\left(S_{j}-\mu\right)}{\sigma}\right]$.

As GEV is a parametric distribution and requires the estimation of only three parameters, few genuine values are sufficient to model the GEV distribution as opposed to the non-parametric techniques which require a much higher number of genuine scores to estimate the distribution reliably. Hence, the parametric techniques have lower computational complexity than the non-parametric techniques.

Given a GEV distribution, estimating the probability that a given score is an outlier is computed from the value of CDF of the GEV distribution. So, the normalization test score vector is computed using the CDF of the GEV distribution, as follows:

$$
S_{i}^{\prime}=G\left(S_{i}, \mu, \sigma, k\right)
$$

where, $S_{i}^{\prime}$ is the $i^{\text {th }}$ class normalized score. After normalization, scores from the different unimodal systems are fused by using a score-level fusion technique. In identification mode, the user is identified if the enrolled subject corresponds to the top score from the fused score vector. Efficiency of the proposed method is compared (see Table 1) with GPD [2] and W-Score [3] methods, using Identification (IR) and Verification (VR) rates. Results are shown over four multimodal biometric datasets: Test Sets I and II are obtained from NIST-BSSR1; while Test Sets III and IV are formed (chimeric) using samples from NIST-BSSR1 and 'FRGC v2.0 + LG4000' datasets respectively.

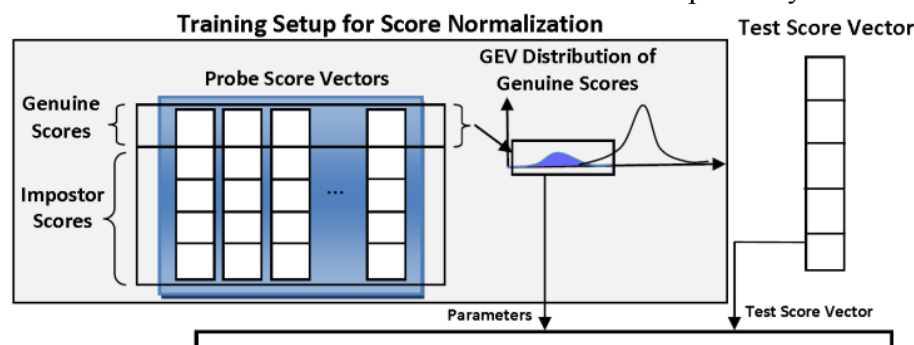

Normalized Score Vector $=$ CDF (Test Score Vector, Parameters)

Figure 1: Architecture of the proposed method for score normalization.

\begin{tabular}{|l|c|c|c|c|c|c|c|c|}
\hline & \multicolumn{2}{|c|}{ Test Set I } & \multicolumn{2}{c|}{ Test Set II } & \multicolumn{2}{c|}{ Test Set III } & \multicolumn{2}{c|}{ Test Set IV } \\
\hline Methods & VR & IR & VR & IR & VR & IR & VR & IR \\
\hline GPD[2] & 94.71 & 81.11 & 95.74 & 89.55 & 94.86 & 82.50 & 98.91 & 97.49 \\
\hline W-Score[3] & 96.75 & 81.75 & 99.80 & 98.06 & 98.23 & 87.03 & 99.35 & 92.73 \\
\hline Our Method & $\mathbf{9 6 . 8 9}$ & $\mathbf{8 5 . 1 1}$ & $\mathbf{9 9 . 9 9}$ & $\mathbf{1 0 0}$ & $\mathbf{9 9 . 8 6}$ & $\mathbf{9 9 . 4 3}$ & $\mathbf{9 9 . 5 9}$ & $\mathbf{9 8 . 0 0}$ \\
\hline
\end{tabular}

Table 1: Identification and verification rate of Test Sets I, II, III, and IV.

[1] A. Jain, K. Nandakumar, A. Ross. Score normalization in multimodal biometric systems. Pattern Recognition, 38(12):2270-2285, 2005.

[2] Z. Shi, F. Kiefer, J. Schneider, and V. Govindaraju. Modeling Biometric Systems Using the General Pareto Distribution (GPD). In SPIE, 6944, 2008.

[3] W. J. Scheirer, A. Rocha, R.J. Micheals, and T. E. Boult. MetaRecognition: The Theory and Practice of Recognition Score Analysis. IEEE Transactions on Pattern Analysis and Machine Intelligence, 33(8):1689-1695, 2011.

[4] S. Kotz and S. Nadarajah. Extreme Value Distributions: Theory and Applications. 1 Edn. World Scientific Publishing Co., 2001. 